\title{
Silence of long non-coding RNA KCNQ10T1 inhibits the proliferation, migration and invasion of hepatocellular carcinoma cells through PI3K/AKT signaling pathway
}

\section{Hongliang Mei}

Wuhan University Zhongnan Hospital

\section{Zhiguo Yu}

Chinese PLA General Hospital

\section{Guanqi Zhang}

Wuhan University Renmin Hospital

\section{Zhiyuan Huang}

Chinese PLA General Hospital

Hanjun Li

Wuhan University Renmin Hospital

Yilin Hu

Chinese PLA General Hospital

Weidong Jin

Chinese PLA General Hospital

Jie Yao

Wuhan University Zhongnan Hospital

Qipin Lu

Chinese PLA General Hospital

Zhisu Liu ( $D$ liuzs53@sina.com )

Wuhan University Zhongnan Hospital

\section{Research}

Keywords: KCNQ10T1, hepatocellular carcinoma, PI3K/AKT signaling pathway, progression

Posted Date: April 15th, 2020

DOl: https://doi.org/10.21203/rs.3.rs-21344/v1

License: (c) (i) This work is licensed under a Creative Commons Attribution 4.0 International License. Read Full License 
Page 2/15 


\section{Abstract}

Background: KCNQ1 opposite strand/antisense transcript 1 (KCNQ10T1) has been reported to be associated with hepatocellular carcinoma (HCC), which is considered as one of the most common cancers worldwide. However, the mechanism of action of KCNQ10T1 in human HCC has not been fully explained. In this study, we aimed to explore the functional role and the potential mechanism of KCNQ10T1 in human HCC.

Methods: First, we analyzed the expression levels of KCNQ10T1 in HCC tissues in starBase database and detected the expression of KCNQ10T1 in HCC cell lines by quantitative real-time polymerase chain reaction assays. Next, we analyzed the role of KCNQ10T1 in migration, invasion and proliferation of HCC by scratch wound healing, transwell and cell counting kit-8 assays. Finally, we analyzed the potential interrelationship between KCNQ10T1 and PI3K/AKT signaling pathway through western blot assays.

Results: Based on bioinformatics analyses, we found that KCNQ10T1 was highly expressed in HCC tissues and its high expression was associated with a poor prognosis in HCC patients. We also confirmed an abnormal increase in the expression of KCNQ10T1 in HCC cell lines. KCNQ10T1 knockdown was found to have a negative impact on proliferation, migration and invasion of HCC cells. In addition, interference with the expression of KCNQ10T1 reduced the phosphorylation level of AKT and the protein level of PI3K, indicating the association of KCNQ10T1 with the PI3K/AKT signaling pathway.

Conclusions: Collectively, this study confirmed the important role of KCNQ10T1 in promoting HCC growth and revealed the inhibitory effect of KCNQ10T1 on the PI3K/AKT signaling pathway. This work may contribute to a better understanding of HCC progression and provide a potential biomarker for HCC.

\section{Background}

Among all kinds of primary liver cancer, hepatocellular carcinoma (HCC) is one of the most common malignant tumors(1). HCC is known for its high invasiveness and mortality. The treatment of patients diagnosed with $\mathrm{HCC}$ is very limited and the five-year survival rate of patients directly decreases due to the transfer of blood and the formation of tumor thrombus(2). Therefore, identification of reliable biomarkers for HCC is particularly important for effective treatment and prognostic improvement of HCC.

Long non-coding RNAs (IncRNAs) are a group of non-coding RNAs more than 200 base pairs(3). LncRNAs were originally thought to be by-products of RNA polymerase transcription and had no biological function. However, immense amounts of studies have recently shown that many IncRNAs have biological functions and are involved in the development of cancers, such as thyroid cancer, breast cancer, gastric cancer, colorectal cancer, lung cancer, and ovarian cancer(4-9). Particularly in HCC, many related IncRNAs are also reported to be involved in its carcinogenesis and progression. For example, the results of Xiao $\mathrm{J}$ et al confirmed that IncRNA HANR is up-regulated in HCC and promotes the development of HCC, which may be a new therapeutic target(10). LncRNA ELM01-AS1 was found to be significantly down-regulated in HCC tissues, and overexpression of ELM01-AS1 in HCC cells inhibited cell proliferation, 
migration and invasion, suggesting its potential as an independent prognostic biomarker for $\mathrm{HCC}(11)$. IncRNA TGLC15 was significantly overexpressed in HCC cell lines and high level of TGLC15 was associated with advanced malignant features such as tumor size and metastasis(12). Despite the identification of numerous IncRNAs associated with HCC, the majority of IncRNAs remain unexplored and their functional roles need to be explained.

KCNQ1 opposite strand/antisense transcript 1 (KCNQ10T1) has been shown to be a chromatin-regulated IncRNA. So far, the abnormal expression and function of KCNQ10T1 have been implicated in some tumors. For example, Li Y et al revealed that IncRNA KCNQ10T1 regulates colon cancer resistance through sponge to miR-34a(13). Feng $\mathrm{W}$ et al found that IncRNA KCNQ10T1 promotes the growth of breast cancer cells by regulating miR-145/CCNE2(14). Zheng L et al found that IncRNA KCNQ10T1 might contribute to the development of non-small cell lung cancer(15). However, its biological function and clinical significance in HCC are still unclear.

The purpose of this study was to explore the function role and the potential mechanism of KCNQ10T1 in HCC. First, we analyzed the expression levels of KCNQ10T1 in HCC tissues in starBase database. Then, we detected the expression of KCNQ10T1 in HCC cell lines by quantitative real-time polymerase chain reaction (qRT-PCR) assays. Next, we analyzed the role of KCNQ10T1 in migration, invasion and proliferation of HCC by scratch wound healing assays, transwell assays and cell counting kit-8 (CCK8) assays. Finally, we analyzed the potential interrelationship between KCNQ10T1 and PI3K/AKT signaling pathway through western blot assays.

\section{Methods}

\section{Bioinformatics analyses}

We analyzed the expression levels of KCNQ10T1 in 374 HCC tissues and 50 normal tissues in starBase (http://starbase.sysu.edu.cn) database. All data was calibrated, standardized and converted using log2. Gene Expression Profiling Interactive Analysis (GEPIA, http://gepia.cancer-pku.cn/) was utilized to figure out the influence of KCNQ10T1 on disease free survival (DFS) in patients with liver cancer.

\section{Cell culture}

The human HCC cell lines (HepG2, SMCC-7721, MHCC-97H) and normal liver cell line (LO2) were purchased from China Center for Type Culture Collection (Wuhan, China). These cell lines were cultured using Dulbecco's Modified Eagle's Medium (DMEM; Thermo Fisher Scientific, Waltham, MA, USA) supplemented with $10 \%$ fetal bovine serum (Gibco, Thermo Fisher Scientific, Waltham, MA, USA), 100 $\mathrm{U} / \mathrm{ml}$ penicillin and $100 \mathrm{ng} / \mathrm{ml}$ streptomycin in a humidified atmosphere of $5 \%(\mathrm{v} / \mathrm{v}) \mathrm{CO}_{2}$ at $37^{\circ} \mathrm{C}$.

\section{RNA extraction and qRT-PCR assay}


Total RNA was extracted from HCC cells by Trizol reagent (Invitrogen, Carlsbad, CA) according to the manufacturer's instructions. Reverse transcription was carried out after total RNA of HCC cells were extracted, according to the instructions of UElris II RT-PCR System for First-Strand cDNA Synthesis (US Everbright ${ }^{\circledR} I n c$, Suzhou, China). SYBR Premix Ex Taq (US Everbright ${ }^{\circledR} I n c$, Suzhou, China) on an ABI 7900 system (Applied Biosystems, Foster City, CA, USA) was employed to conduct qRT-PCR assay using glyceraldehyde 3-phosphate dehydrogenase (GAPDH) as endogenous control. The $2^{-\triangle \Delta C t}$ method was employed to detect the comparative quantification. The primer was purchased from Sangon Biotech (Shanghai, China) and its sequences were as follows:

KCNQ10T1 forward, 5'-CTTTGCAGCAACCTCCTTGT-3'; KCNQ10T1 reverse, 5'TGGGGTGAGGGATCTGAA-3'; GAPDH forward, 5'- ACGGATTTGGTCGTATTGGGCG-3'; GAPDH reverse, 5'GCTCCTGGAAGATGGTGATGGG-3'.

\section{Cell transfection}

KCNQ10T1-specific small interfering RNA (siRNA-KCNQ10T1, the sequence is GCCAAUAGCAACUGACUAA) was designed and synthesized by Ribobio (Guangzhou, China). Negative control (NC) and siRNA-KCNQ10T1 were separately transfected into HepG2, SMMC-7721 and MHCC-97H cells using Lipofectamine 2000 transfection reagent (Invitrogen; Thermo Fisher Scientific, Waltham, MA, USA) according to the manufacturer's protocol. Cells were collected and ready for the following assays after $48 \mathrm{~h}$ transfection. Transfection efficiency was evaluated by qRT-PCR assay.

\section{Scratch wound healing assay}

The scratch wound healing assay was used to evaluate the transverse migratory ability of transfected cells. Briefly, cells were plated on 6-well plates and scraped by a $10 \mu$ lip to generate uniform wounds prior to transfection (siRNA-KCNQ10T1 or siRNA-NC). The wells were washed three times with phosphatebuffered saline (PBS) to remove the exfoliated cells, and then medium with $2 \%$ fetal bovine serum was added. The cells were further incubated in a $37^{\circ} \mathrm{C}$ and $5 \% \mathrm{CO}_{2}$ incubator. The photos at a magnification of $40 \times$ were taken at 0,24 , and $48 \mathrm{~h}$, respectively.

\section{CCK8 assay}

HepG2, SMMC-7721 and MHCC-97H cells were respectively plated into 96-well plates and cultured for around 17 24 $\mathrm{h}$ and then were transfected with siRNA-KCNQ10T1 or NC using Lipofectamine 2000 according to the manufacturer's protocol. The cells were separately incubated for $0,24,48$ and $72 \mathrm{~h}$. CCK8 solution (Beyotime Institute of Biotechnology, Shanghai, China) was added and incubated for $1 \mathrm{~h}$. The absorbance at $450 \mathrm{~nm}$ was measured using a Multiskan FC (Thermo Fisher Scientific, Waltham, MA, USA).

\section{Transwell assay}


Transwell chambers uncoated or coated with Matrigel (BD Biosciences, USA) were employed to assess cell vertical migratory or invasive abilities, respectively. In brief, cells transfected with siRNA-KCNQ10T1 or NC were digested to a concentration of $1 \times 10^{5} \mathrm{cells} / \mathrm{ml}$ and seeded in the upper chamber with serumfree medium. At the same time, $600 \mu 10 \%$ FBS-DMEM was added to the lower layer. After $24 \mathrm{~h}$, the upper device was secured and washed by PBS carefully and then fixed with $4 \%$ polyformaldehyde for 20 min at room temperature. Then the chamber was stained in a 24-well plate containing $600 \mu$ l of crystal violet for $20 \mathrm{~min}$. The stained cells were then observed under a microscope at a magnification of $100 x$.

\section{Western blot assay}

Total proteins were extracted from HCC cells after $48 \mathrm{~h}$ transfection using cell lysis buffer (Beyotime Biotechnology Shanghai, China). Protein samples were electrophoresed on $12 \%$ sodium dodecyl sulfate polyacrylamide gel electrophoresis (SDS-PAGE) to resolve, and then transferred to polyvinylidene fluoride membrane. The membrane was blocked with $5 \%$ BSA at room temperature for $60 \mathrm{~min}$. After incubated with primary antibodies $\mathrm{PI} 3 \mathrm{~K}$ and p-AKT (1:1000, ABclonal) at $4^{\circ} \mathrm{C}$ overnight and secondary antibodies (1:10000, ABclonal) for 90 min at room temperature, ECL reagents (Millpore, USA) were used to detect the signal of target proteins. The images of the gels were scanned using Bio-Rad Gel Doc XR+ system (BioRad, Hercules, CA, USA). GAPDH was used as an internal control.

\section{Statistical analysis}

Continuous data were presented as the mean values \pm SD. All statistical analyses were carried out using GraphPad Prism 7.0 (La Jolla, CA, USA). Student's t test was used to compare the differences between two dependent groups. $P<0.05$ was considered to be statistically significant.

\section{Results}

\section{KCNQ10T1 was evidently up-regulated and associated with prognosis in HCC patients}

According to starBase database, the expression of KCNQ10T1 in HCC was higher than that in normal (Figure 1A, $P<0.001$ ). To verify this result, three human HCC cell lines (namely HepG2, SMCC-7721 and MHCC-97H) and one normal liver cell line LO2 were used to detect the expression of KCNQ10T1 by qRTPCR assays. Results showed that KCNQ10T1 was highly expressed in HCC cells compared to normal cells (Figure 1B, $P<0.05, P<0.01$ ). Through GEPIA, we found that KCNQ10T1 had a certain effect on the DFS of patients with HCC. Before about 55 months, patients with high KCNQ10T1 expression had lower survival rate and poor prognosis (Figure 1C). Taken together, these results unearthed that KCNQ10T1 was evidently up-regulated in HCC cells and tissues, and its high expression was associated with low survival rate and poor prognosis for HCC patients.

We examined the transverse migration ability of HCC cells by scratch wound healing assays in HepG2, SMCC-7721, and MHCC-97H cell lines. The results showed that the cell healing rate of the cells transfected siRNA-KCNQ10T1 was slower than that of the NC group in the HepG2 cell line (Figure 2B, 
$P<0.05$ ). We obtained the same results in the experiments of SMCC-7721 and MHCC-97H cell lines (Figure 2B, $P<0.01)$. These results indicated that KCNQ10T1 knockdown inhibited the transverse migration of HCC cells.

The transwell assay was used to investigate whether KCNQ10T1 was involved in the vertical migration of HCC cells. We found that vertical mobility of KCNQ10T1 knockdown in the HepG2 cell line was significantly lower than that of the NC group (Figure 2C, $P<0.01$ ). We obtained similar results in the transwell assays of SMCC-7721 and MHCC-97H cell lines (Figure $2 \mathrm{C}, P<0.05, P<0.01$ ). Thus, KCNQ10T1 knockdown inhibited the cell vertical migration ability of HCC. Taken together, these findings indicated that KCNQ10T1 knockdown suppressed migration of HCC cells.

\section{KCNQ10T1 knockdown inhibited HCC proliferation and invasion}

The results of CCK8 assay showed that KCNQ10T1 knockdown cells were less proliferative in HepG2 cell lines than control cells (Figure 3A, $P<0.01$ ). We obtained similar results in SMCC-7721 and MHCC-97H cell lines (Figure 3A, $P<0.01$ ). Therefore, KCNQ10T1 knockdown could inhibit HCC cell proliferation.

Through the invasion experiments, we found that the invasive rates of KCNQ10T1 knockdown in HepG2/SMCC-7721/MHCC-97H cell lines were all lower than that in NC group (Figure $3 B, P<0.05, P<0.01$ ). These results suggested that knockdown of KCNQ10T1 inhibited the invasion of HCC cells.

\section{KCNQ10T1 knockdown suppressed PI3K/AKT pathway}

Studies have shown that PI3K/AKT signaling pathway is closely related to the regulatory mechanism of IncRNAs in HCC(16-18). We wondered whether KCNQ10T1 could affect the PI3K/AKT signaling pathway in HCC. Therefore, siRNA-KCNQ10T1 was transfected into the HepG2/SMCC-7721/MHCC-97H cell lines to study the effect of KCNQ10T1 knockdown on the PI3K/AKT signaling pathway. As shown in the Figure 4, KCNQ10T1 knockdown in HepG2 cell line reduced the expression of PI3K and p-AKT compared to the NC group. In the SMCC-7721/MHCC-97H cell lines, we obtained the same results by western blot analysis. Collectively, these results revealed that KCNQ10T1 could suppress the PI3K/AKT signaling pathway in HCC cells.

\section{Discussion}

Growing evidence indicates that IncRNAs may play a critical role in the pathophysiology of human diseases as an oncogene or tumor suppressor gene, especially in the occurrence and development of tumors $(19,20)$. Numerous studies have reported that IncRNA KCNQ10T1 is involved in various diseases, such as non-small cell lung cancer, breast cancer cells, cholangiocarcinoma(14, 21, 22). Moreover, Li et al found KCNQ10T1 mediates the growth of HCC(23). However, the functional role of KCNQ10T1 in human HCC remains to be further explored.

In this study, based on the data from starBase database, we found that KCNQ10T1 was up-regulated in HCC tissues. Consistent with our findings, the result of qRT-PCR assay demonstrated that KCNQ10T1 had 
higher expression levels in three different kinds of HCC cell lines compared with the normal control group. This result is consistent with previous report that the expression of KCNQ10T1 was unusually elevated in HCC tissues(23). Those results suggested that KCNQ1OT1 might participate in the occurrence and progression of HCC. Furthermore, we conducted the DFS analysis of KCNQ10T1 in HCC through GEPIA. Significantly, the high expression of KCNQ10T1 had a poor prognosis, while low expression of KCNQ10T1 resulted in a better prognosis. As Li et al exhibited, HCC patients with a high KCNQ1OT1 expression experienced a worse overall and DFS times(23). Besides, up-regulation of KCNQ10T1 might mean shorter survival of colon adenocarcinoma(24).

Based on the above analyses, we predicted that KCNQ10T1 may play an important functional role in HCC. To validate this prediction, a series of assays were conducted, including scratch wound healing, CCK8 and transwell assays. The outcome showed that KCNQ10T1 knockdown suppressed the migration, proliferation and invasion of HCC cells in HepG2/SMCC-7721/MHCC-97H cell lines. Through literature studies, we found that KCNQ10T1 mediates the growth of HCC cells, and knockdown of KCNQ10T1 significantly inhibits the viability of HCC cells(23). Additionally, KCNQ10T1 has been reported to participate in cell proliferation in various cancers, including breast cancer, melanoma, and tongue cancer(25-27). Moreover, KCNQ10T1 facilitates cell migration in colorectal cancer(28).

As previous research presented, PI3K/AKT signaling pathway is closely correlated to HCC progression(29, 30), and IncRNAs facilitate cell growth and metastasis by PI3K/AKT signaling pathway in HCC(31). In order to explore the effect of KCNQ10T1 on PI3K/AKT signaling pathway, western blot analysis was conducted in our study. Interestingly, we discovered that KCNQ10T1 knockdown reduced the phosphorylation level of AKT and the protein level of PI3K. These findings suggested that KCNQ10T1 might promote HCC growth by regulating PI3K/AKT signaling pathway.

\section{Conclusions}

In summary, these data indicated that silence of KCNQ10T1 could inhibit the proliferation, migration and invasion in human HCC via suppressing the PI3K/AKT signaling pathway. This work not only contributes to a better understanding of the mechanism of KCNQ10T1 in HCC, but also indicates that KCNQ10T1 might serve as a promising biomarker in HCC. Despite these promising results, limitations remain. The expression profile of KCNQ10T1 and its association with HCC prognosis are tentatively absent due to the limited clinical resources collected. In addition, the detailed molecular mechanism of KCNQ10T1 in HCC remains to be explored.

\section{Abbreviations}

HCC, hepatocellular carcinoma; IncRNA, long non-coding RNA; KCNQ10T1, KCNQ1 opposite strand/antisense transcript 1; qRT-PCR, quantitative real-time polymerase chain reaction; CCK8, cell counting kit-8; GEPIA, Gene Expression Profiling Interactive Analysis; DFS, disease free survival; siRNA, 
small interfering RNA; NC, negative control; DMEM, Dulbecco's Modified Eagle's Medium; PBS, phosphatebuffered saline.

\section{Declarations}

\section{Ethics approval and consent to participate}

Not applicable.

\section{Consent for publication}

Not applicable.

\section{Availability of data and materials}

The datasets used and/or analyzed during the current study are available from the corresponding author on reasonable request.

\section{Competing interests}

The authors declare that they have no competing interests.

\section{Funding}

Not applicable.

\section{Authors' contributions}

HM and ZY conceived the study, performed the experiments, and made writing-original draft preparation. $\mathrm{GZ}, \mathrm{ZH}$ and HL provided insight and participated in data analysis. YH, WJ and JY helped with the writing/review of the manuscript. ZL and QL contributed to project administration and supervision. All authors read and approved the final manuscript.

\section{Acknowledgements}

We thank the technical support and guidance provided by Wuhan Bojie Biomedical Science and Technology CO., LTD.

\section{References}

1. Mittal S, El-Serag HB. Epidemiology of hepatocellular carcinoma: consider the population. Journal of clinical gastroenterology. 2013;47 Suppl:S2-6.

2. Kinoshita A, Koike K, Nishino H. Clinical features and prognosis of elderly patients with hepatocellular carcinoma not indicated for surgical resection. Geriatrics \& gerontology international. 2017;17(2):189-201. 
3. Guttman M, Amit I, Garber M, French C, Lin MF, Feldser D, et al. Chromatin signature reveals over a thousand highly conserved large non-coding RNAs in mammals. Nature. 2009;458(7235):223-7.

4. Chen $Y, Y u X, X u Y$, Shen $H$. Identification of dysregulated IncRNAs profiling and metastasisassociated IncRNAs in colorectal cancer by genome-wide analysis. Cancer medicine. 2017;6(10):2321-30.

5. Gou L, Zou H, Li B. Long noncoding RNA MALAT1 knockdown inhibits progression of anaplastic thyroid carcinoma by regulating miR-200a-3p/FOXA1. Cancer biology \& therapy. 2019:1-11.

6. Gu Y, Chen T, Li G, Yu X, Lu Y, Wang H, et al. LncRNAs: emerging biomarkers in gastric cancer. Future oncology (London, England). 2015;11(17):2427-41.

7. Lei $H, G a o Y, X u X$. LncRNA TUG1 influences papillary thyroid cancer cell proliferation, migration and EMT formation through targeting miR-145. Acta biochimica et biophysica Sinica. 2017;49(7):588-97.

8. Nagini S. Breast Cancer: Current Molecular Therapeutic Targets and New Players. Anti-cancer agents in medicinal chemistry. 2017;17(2):152-63.

9. Zhang R, Xia Y, Wang Z, Zheng J, Chen Y, Li X, et al. Serum long non coding RNA MALAT-1 protected by exosomes is up-regulated and promotes cell proliferation and migration in non-small cell lung cancer. Biochemical and biophysical research communications. 2017;490(2):406-14.

10. Xiao J, Lv Y, Jin F, Liu Y, Ma Y, Xiong Y, et al. LncRNA HANR Promotes Tumorigenesis and Increase of Chemoresistance in Hepatocellular Carcinoma. Cellular physiology and biochemistry : international journal of experimental cellular physiology, biochemistry, and pharmacology. 2017;43(5):1926-38.

11. Luo T, Chen M, Zhao Y, Wang D, Liu J, Chen J, et al. Macrophage-associated IncRNA ELMO1-AS1: a novel therapeutic target and prognostic biomarker for hepatocellular carcinoma. OncoTargets and therapy. 2019;12:6203-16.

12. Chen $Y$, Huang F, Deng L, Tang Y, Li D, Wang T, et al. Long non-coding RNA TGLC15 advances hepatocellular carcinoma by stabilizing Sox4. Journal of clinical laboratory analysis. 2019:e23009.

13. Li Y, Li C, Li D, Yang L, Jin J, Zhang B. IncRNA KCNQ10T1 enhances the chemoresistance of oxaliplatin in colon cancer by targeting the miR-34a/ATG4B pathway. OncoTargets and therapy. 2019;12:2649-60.

14. Feng W, Wang C, Liang C, Yang H, Chen D, Yu X, et al. The Dysregulated Expression of KCNQ10T1 and Its Interaction with Downstream Factors miR-145/CCNE2 in Breast Cancer Cells. Cellular physiology and biochemistry : international journal of experimental cellular physiology, biochemistry, and pharmacology. 2018;49(2):432-46.

15. Zheng L, Zhang FX, Wang LL, Hu HL, Lian YD. LncRNA KCNQ10T1 is overexpressed in non-small cell lung cancer and its expression level is related to clinicopathology. European review for medical and pharmacological sciences. 2019;23(16):6944-50.

16. Lee YR, Chen M, Pandolfi PP. The functions and regulation of the PTEN tumour suppressor: new modes and prospects. Nature reviews Molecular cell biology. 2018;19(9):547-62.

17. He B, Peng F, Li W, Jiang Y. Interaction of IncRNA-MALAT1 and miR-124 regulates HBx-induced cancer stem cell properties in HepG2 through PI3K/Akt signaling. Journal of cellular biochemistry. 
2019;120(3):2908-18.

18. Li Y, Ye Y, Feng B, Qi Y. Long Noncoding RNA IncARSR Promotes Doxorubicin Resistance in Hepatocellular Carcinoma via Modulating PTEN-PI3K/Akt Pathway. Journal of cellular biochemistry. 2017;118(12):4498-507.

19. Li J, Li Z, Zheng W, Li X, Wang Z, Cui Y, et al. LncRNA-ATB: An indispensable cancer-related long noncoding RNA. Cell proliferation. 2017;50(6).

20. Bhan A, Soleimani M, Mandal SS. Long Noncoding RNA and Cancer: A New Paradigm. Cancer research. 2017;77(15):3965-81.

21. Sun X, Xin Y, Wang M, Li S, Miao S, Xuan Y, et al. Overexpression of long non-coding RNA KCNQ10T1 is related to good prognosis via inhibiting cell proliferation in non-small cell lung cancer. Thoracic cancer. 2018;9(5):523-31.

22. Sun H, Li Y, Kong H, Dai S, Qian H. Dysregulation of KCNQ10T1 promotes cholangiocarcinoma progression via miR-140-5p/SOX4 axis. Archives of biochemistry and biophysics. 2018;658:7-15.

23. Li C, Miao R, Zhang J, Qu K, Liu C. Long non-coding RNA KCNQ10T1 mediates the growth of hepatocellular carcinoma by functioning as a competing endogenous RNA of miR-504. International journal of oncology. 2018.

24. Zhang K, Yan J, Yi B, Rui Y, Hu H. High KCNQ10T1 expression might independently predict shorter survival of colon adenocarcinoma. Future oncology (London, England). 2019;15(10):1085-95.

25. Zhang Z, Weaver DL, Olsen D, deKay J, Peng Z, Ashikaga T, et al. Long non-coding RNA chromogenic in situ hybridisation signal pattern correlation with breast tumour pathology. Journal of clinical pathology. 2016;69(1):76-81.

26. Guo B, Zhang Q, Wang H, Chang P, Tao K. KCNQ10T1 promotes melanoma growth and metastasis. Aging. 2018;10(4):632-44.

27. Zhang S, Ma H, Zhang D, Xie S, Wang W, Li Q, et al. LncRNA KCNQ10T1 regulates proliferation and cisplatin resistance in tongue cancer via miR-211-5p mediated Ezrin/Fak/Src signaling. Cell death \& disease. 2018;9(7):742.

28. Bian Y, Gao G, Zhang Q, Qian H, Yu L, Yao N, et al. KCNQ10T1/miR-217/ZEB1 feedback loop facilitates cell migration and epithelial-mesenchymal transition in colorectal cancer. Cancer biology \& therapy. 2019;20(6):886-96.

29. Jiang H, Zhou Z, Jin S, Xu K, Zhang H, Xu J, et al. PRMT9 promotes hepatocellular carcinoma invasion and metastasis via activating PI3K/Akt/GSK-3beta/Snail signaling. Cancer science. 2018;109(5):1414-27.

30. Chang M, Wu M, Li H. Curcumin combined with glycyrrhetinic acid inhibits the development of hepatocellular carcinoma cells by down-regulating the PTEN/PI3K/AKT signalling pathway. American journal of translational research. 2017;9(12):5567-75.

31. Huang JL, Cao SW, Ou QS, Yang B, Zheng SH, Tang J, et al. The long non-coding RNA PTTG3P promotes cell growth and metastasis via up-regulating PTTG1 and activating PI3K/AKT signaling in hepatocellular carcinoma. Molecular cancer. 2018;17(1):93. 

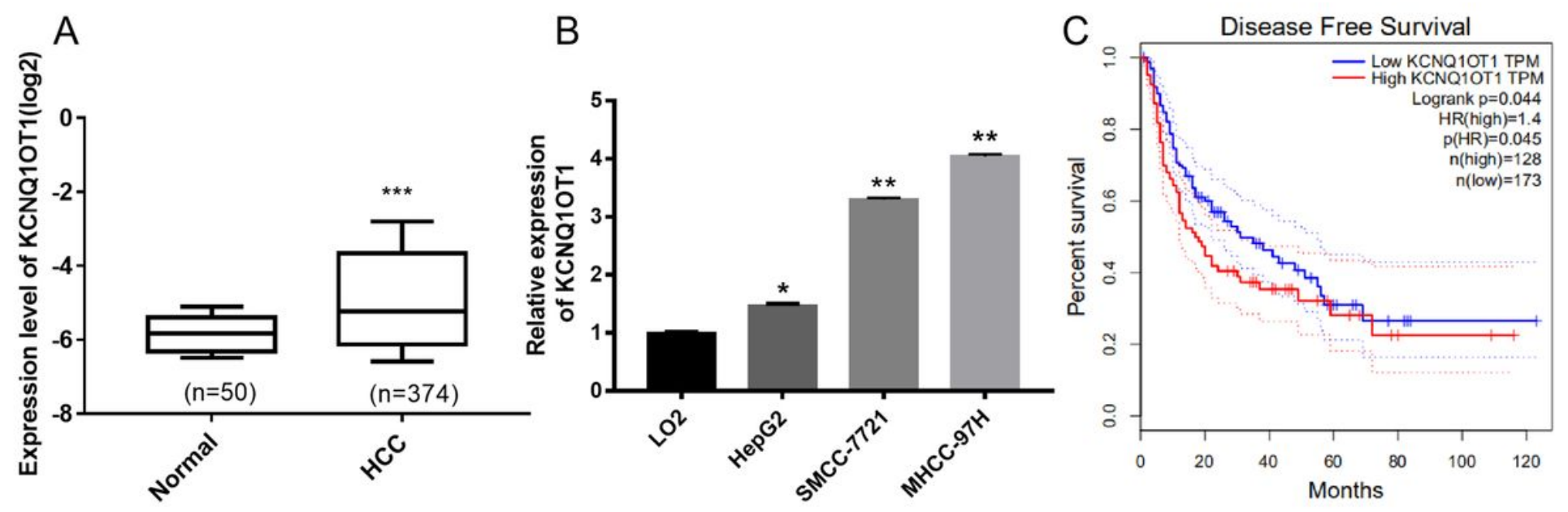

Figure 1

Expression and survival analysis of KCNQ10T1. Notes: (A) The expression levels of KCNQ10T1 in 374 HCC tissues and 50 normal tissues as analyzed by starBase database. (B) The expression levels of KCNQ10T1 in the normal and three HCC cell lines as determined by qRT-PCR assays. (C) DFS of KCNQ10T1 in HCC tissues by GEPIA. *, $\mathrm{P}<0.05$; **, $\mathrm{P}<0.01$; ***, $\mathrm{P}<0.001$. Abbreviations: KCNQ10T1, KCNQ1 opposite strand/antisense transcript 1; qRT-PCR, quantitative reverse transcription polymerase chain reaction; HCC, hepatocellular carcinoma; DFS, disease free survival ; GEPIA, Gene Expression Profiling Interactive Analysis. 

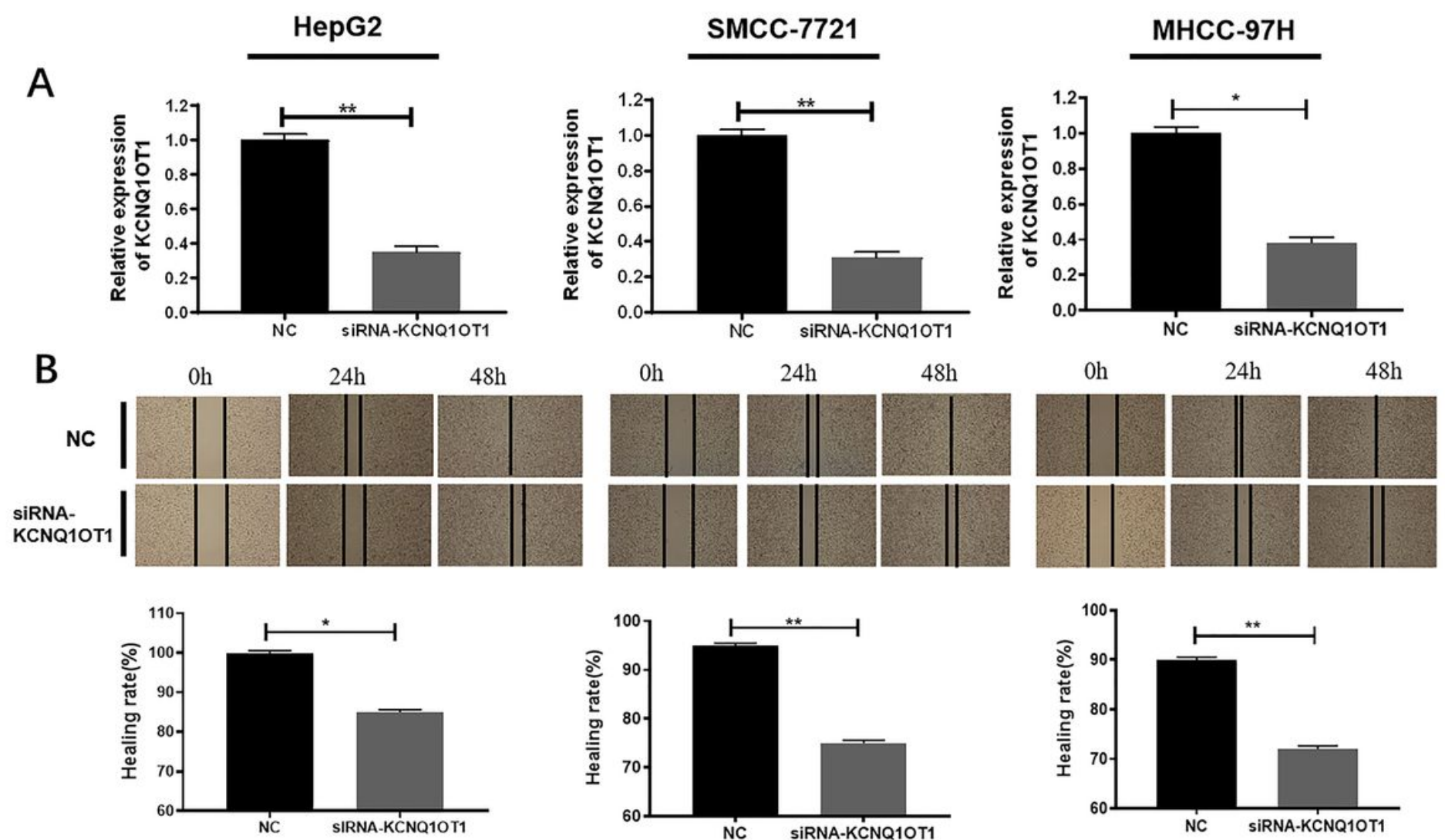

C
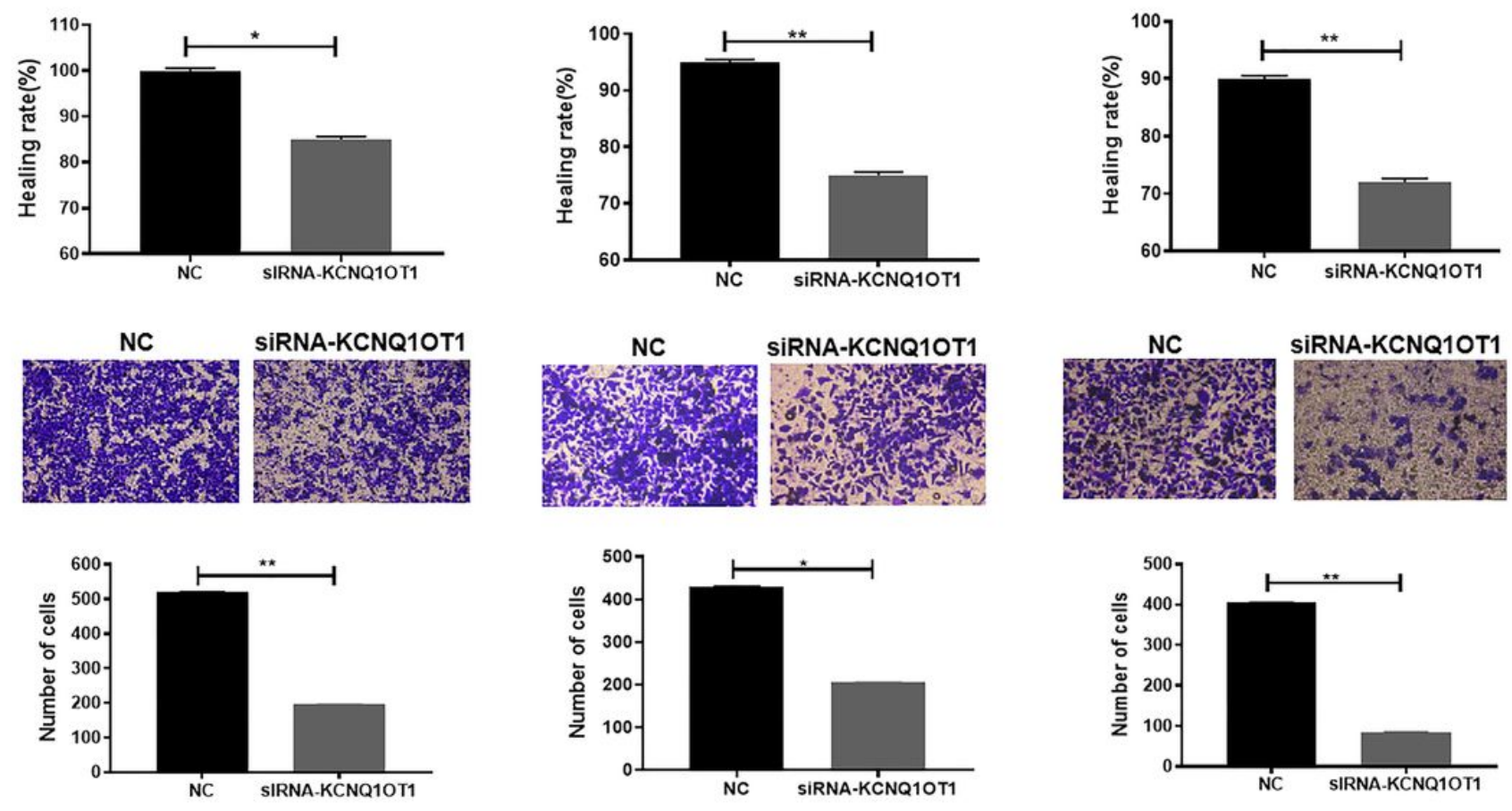

Figure 2

KCNQ10T1 knockdown inhibited migration of HCC cells. Notes: (A) The interference efficiency of siRNAKCNQ10T1 in HepG2/SMCC-7721/ MHCC-97H as analyzed by qRT-PCR assays. (B) The transverse migration of NC cells and KCNQ10T1 knockdown cells in HepG2/ SMCC-7721/ MHCC-97H was determined by scratch wound healing assays at a magnification of 40x. (C) The vertical migration of NC cells and KCNQ10T1 knockdown cells in HepG2/ SMCC-7721/ MHCC-97H was measured by transwell assays at a magnification of $100 \times .{ }^{*}, \mathrm{P}<0.05 ; * \star, P<0.01$. Abbreviations: $\mathrm{HCC}$, hepatocellular carcinoma; siRNA, small interfering RNA; KCNQ10T1, KCNQ1 opposite strand/antisense transcript 1; qRT-PCR, quantitative reverse transcription polymerase chain reaction; $\mathrm{NC}$, negative controls. 

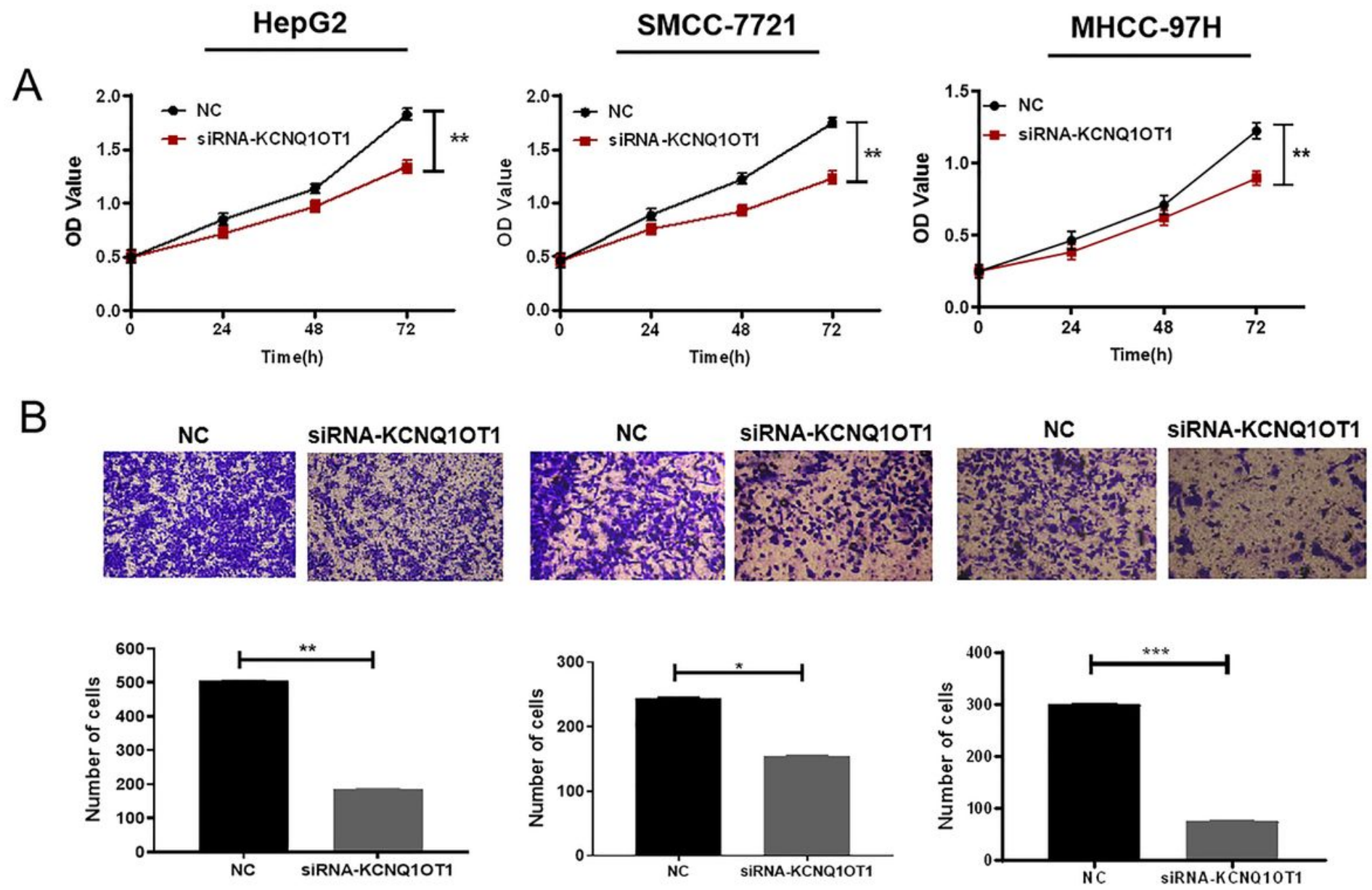

\section{Figure 3}

KCNQ10T1 knockdown inhibited proliferation and invasion of HCC cells. Notes: (A) The proliferation of NC cells and KCNQ10T1 knockdown cells in HepG2/ SMCC-7721/ MHCC-97H was determined by CCK8 assays. (B) The invasion of NC cells and KCNQ10T1 knockdown cells in HepG2/ SMCC-7721/ MHCC$97 \mathrm{H}$ was measured by transwell assays at a magnification of $100 \times$. ${ }^{*}, \mathrm{P}<0.05 ; * \star, P<0.01 ; * \star \star, P<0.001$. Abbreviations: KCNQ10T1NC, KCNQ1 opposite strand/antisense transcript 1; NC, negative controls; HCC, hepatocellular carcinoma; CCK8, cell counting kit-8.

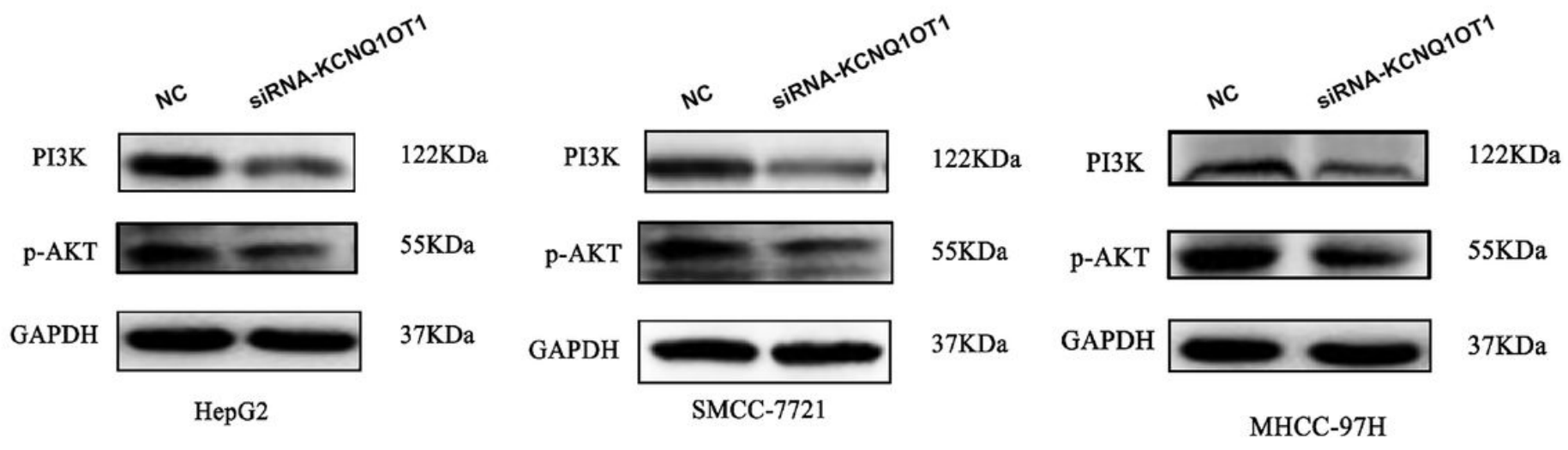


Figure 4

Effects of KCNQ10T1 on PI3K/AKT signaling pathway in HCC cells. Notes: Western blot analysis of the expression of PI3K, GAPDH and phosphorylated AKT in NC cells and KCNQ10T1 knockdown cells in HepG2/ SMCC-7721/ MHCC-97H. Abbreviations: KCNQ1OT1NC, KCNQ1 opposite strand/antisense transcript 1; NC, negative controls; HCC, hepatocellular carcinoma. 